\title{
Surface Chemical Functionalization of Wrinkled Thiol-ene Elastomers for Promoting Cellular Alignment
}

Stephen J. Ma ${ }^{a, t}$, Eden M. Ford ${ }^{a, t}$, Lisa A. Sawicki ${ }^{a}$, Bryan P. Sutherland ${ }^{b}$, Nicole I. Halaszynski ${ }^{b}$, Benjamin J. Carberry ${ }^{a}$, Norman J. Wagner ${ }^{a, c}$, April M. Kloxin $^{a, b^{*}}$, Christopher J. Kloxin $^{a, b^{*}}$

a) Department of Chemical and Biomolecular Engineering, 150 Academy Street, Newark, DE 19716

b) Department of Materials Science and Engineering, 201 DuPont Hall, Newark, DE 19716

c) Center for Molecular and Engineering Thermodynamics, 150 Academy Street, Newark, DE 19716

*Corresponding Authors:

Email: cjk@udel.edu, akloxin@udel.edu 


\section{Table of Contents}

Figure S1 Custom-built stretching stage for straining elastomers.

Figure S2 ESI MS of AF647-RGDS peptide.

Figure S3 $\quad{ }^{1} \mathrm{H}$ NMR Spectra of lithium phenyl-2,4,6-trimethylbenzoylphosphinate (LAP) photoinitiator in $\mathrm{D}_{2} \mathrm{O}$

Figure S4 Conversion data of thiol and ene during elastomer formation

Figure S5 Dark polymerization observed in the free radical polymerization

Figure S6 XPS spectra of fluorine peak area versus PFDT solution concentration

Figure S7 Static Contact angle measurements on functionalized elastomer surfaces

Figure S8 Wavelength and amplitude measurements via confocal microscopy

Figure S9 Confocal images of wrinkles photopatterned with fluorescent probe.

Figure S10 Consistency of amplitude and wavelength across a single sample and between replicates

Figure S11 Protocol for determining cellular alignment of hMSCs on wrinkled substrates

Table S1 Statistical analysis of hMSC attachment in response to surface functionalization (to 3 significant figures).

Table S2 Statistical analysis of hMSC attachment with increasing wrinkle wavelength (to 3 significant figures)

Table S3 Statistical analysis of hMSC nuclear alignment with increasing wrinkle wavelength (to 3 significant figures) 


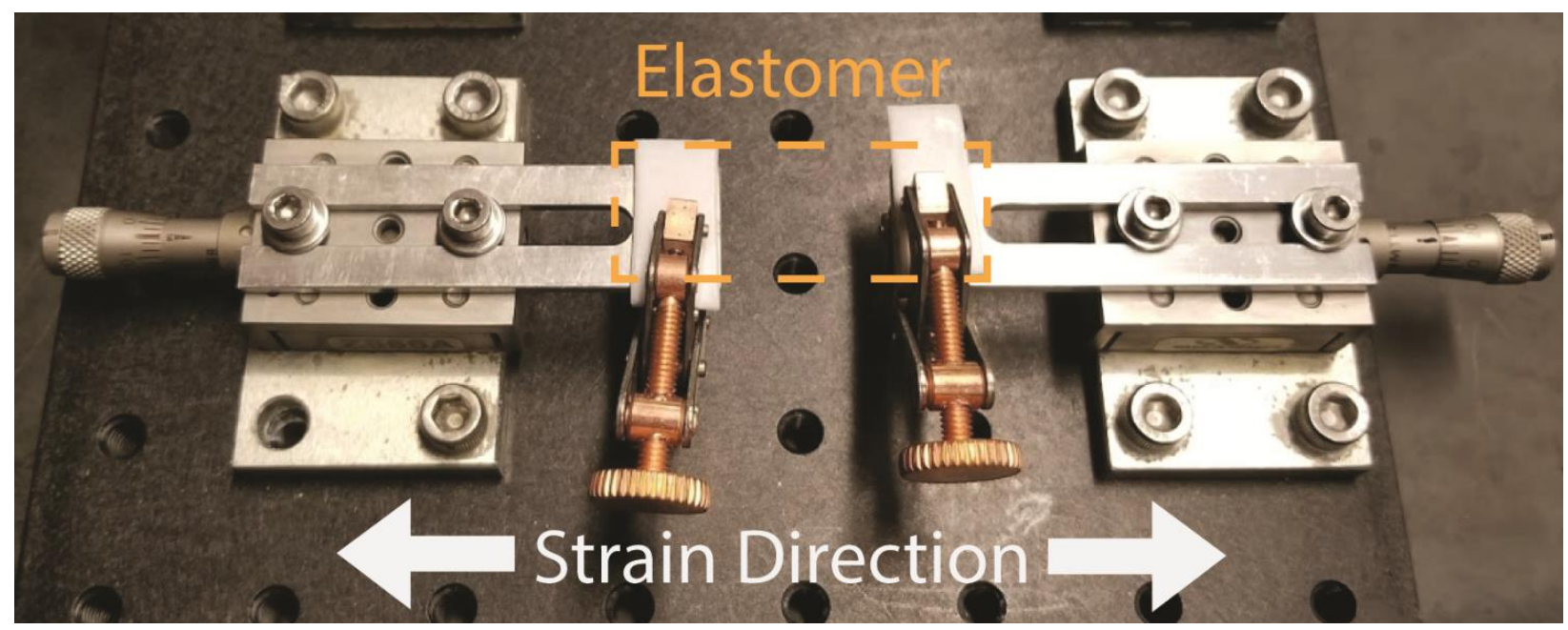

Figure S1. Custom-built stretching stage for straining elastomers. Linear stages (UMR3.5), with attached BM11.5 micrometers (Newport) were mounted on an optical bread board. Aluminum brackets were machined and screwed into the linear stage such that the two brackets were separated by $25 \mathrm{~mm}$ at the center of the set up. Elastomers were mounted on these brackets, sandwiched with Teflon sheets, and clamped down at the ends. Strain was applied by turning the micrometer to the desired length. This entire optical breadboard set up, including the mounted elastomer, can be submerged under water to transfer polymer thin films from a silicon wafer backing onto the strained elastomer.

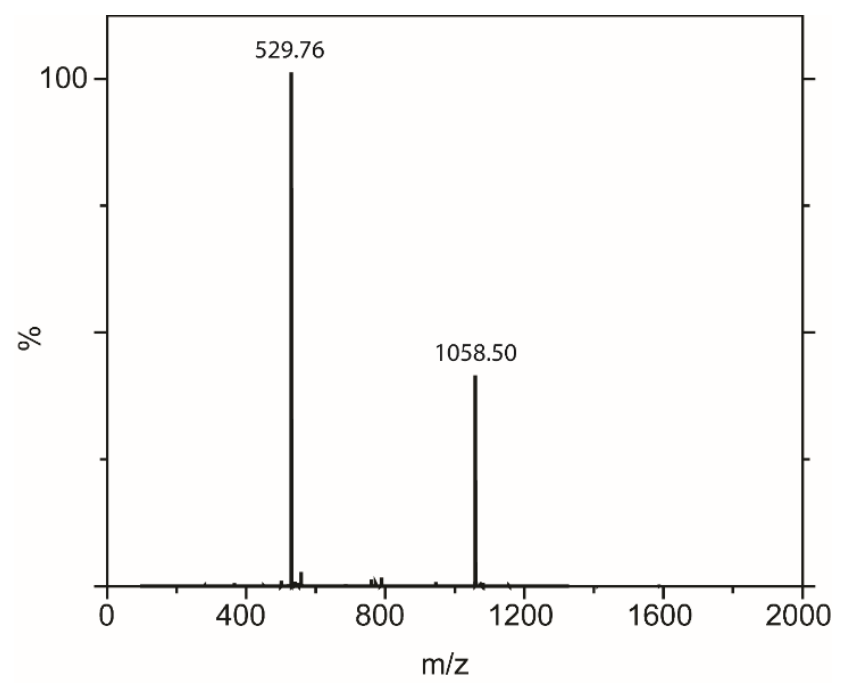

Figure S2. ESI MS of AF647-RGDS peptide. Calculated mass: $[\mathrm{m}+1, \mathrm{H}+]=1059 \mathrm{Da},[\mathrm{m}+2 \mathrm{H}+]=1060 \mathrm{Da}$. Measured $[\mathrm{m}+1, \mathrm{H}+]=$ 1058.5 Da, $[\mathrm{m}+2 \mathrm{H}+]=529.76 \times 2=1059.52 \mathrm{Da}$ 


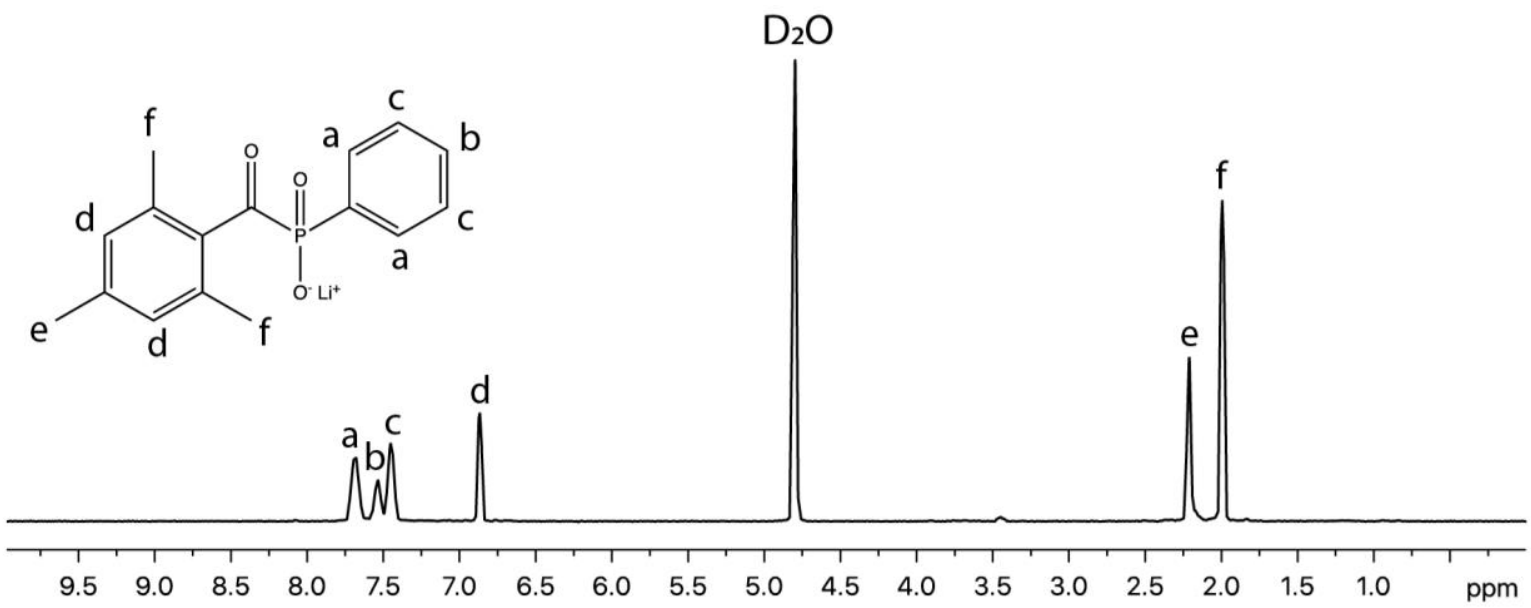

Figure S3. ${ }^{1} \mathrm{H}$ NMR Spectra of lithium phenyl-2,4,6-trimethylbenzoylphosphinate (LAP) photoinitiator in $\mathrm{D}_{\mathbf{2}} \mathrm{O}$. Peaks identified on the NMR spectra correspond to the hydrogens indicated on the LAP chemical structure.

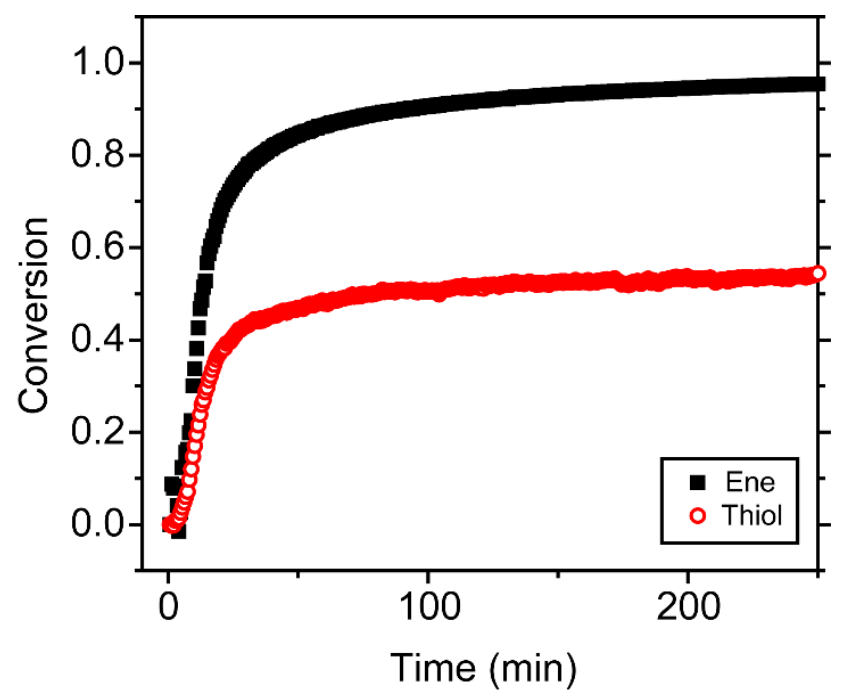

Figure S4. Conversion data of thiol and ene during elastomer formation. In situ FTIR measurements of the 1:2 PETMP:TEGDA system confirms quantitative conversion of thiol and roughly $50 \%$ conversion of the acrylates. 


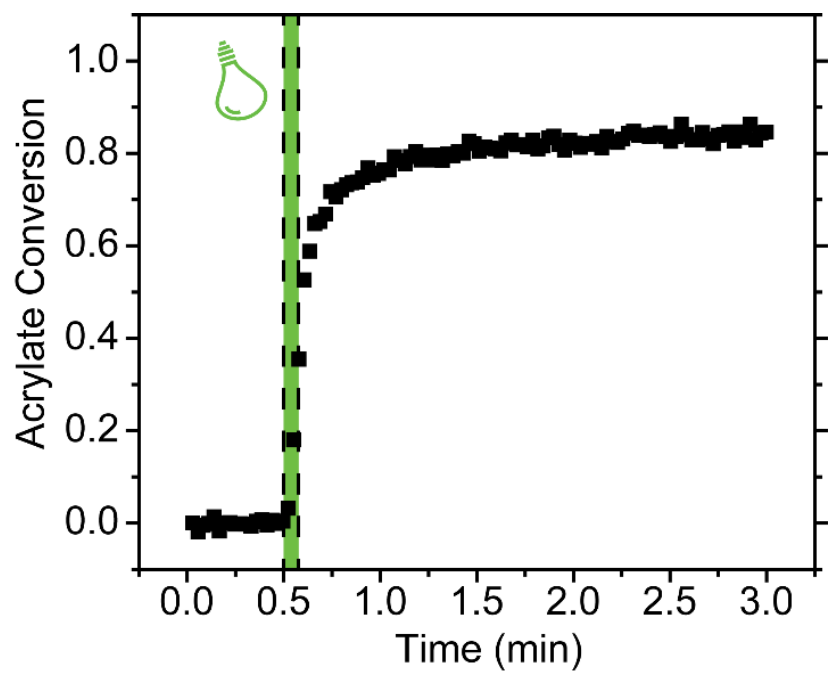

Figure S5. Dark polymerization observed in the free radical polymerization. Despite irradiating the thin film elastomer substrate for only 5 seconds (green highlighted region), acrylate conversion continues to rise for another 30 seconds before hitting a plateau conversion. This dark polymerization is likely due to diffusion limited radical termination. 


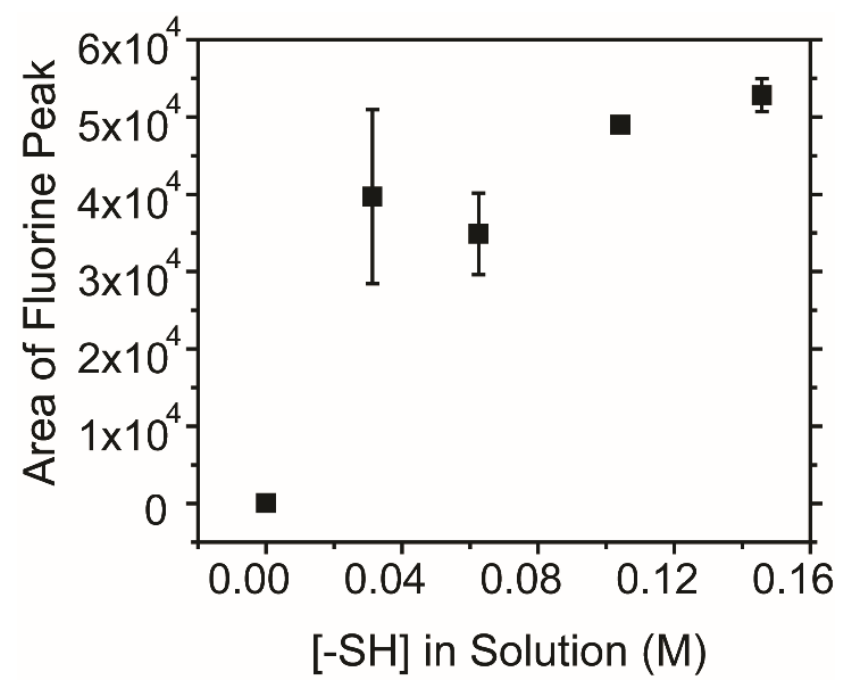

Figure S6. XPS spectra of fluorine peak area versus PFDT solution concentration. X-ray photoelectron spectroscopy (XPS) experiments performed on a surface functionalized with a monofunctionalthiol, perfluorodecanethiol (PFDT), revealed that PFDT concentrations as low at $15 \mathrm{mg} / \mathrm{mL}(0.31 \mathrm{M})$ in solvent were sufficient to approach the saturation limit at the polymer surface. Thus, using PETMP (MW $=488 \mathrm{~g} / \mathrm{mol}$ ), which has four times the number of thiols as the PFDT (MW $=480 \mathrm{~g} / \mathrm{mol}$ ) at the same mass concentration (i.e., $15 \mathrm{mg} / \mathrm{mL}$ ), is sufficient to create a thiol-rich elastomer surface. 


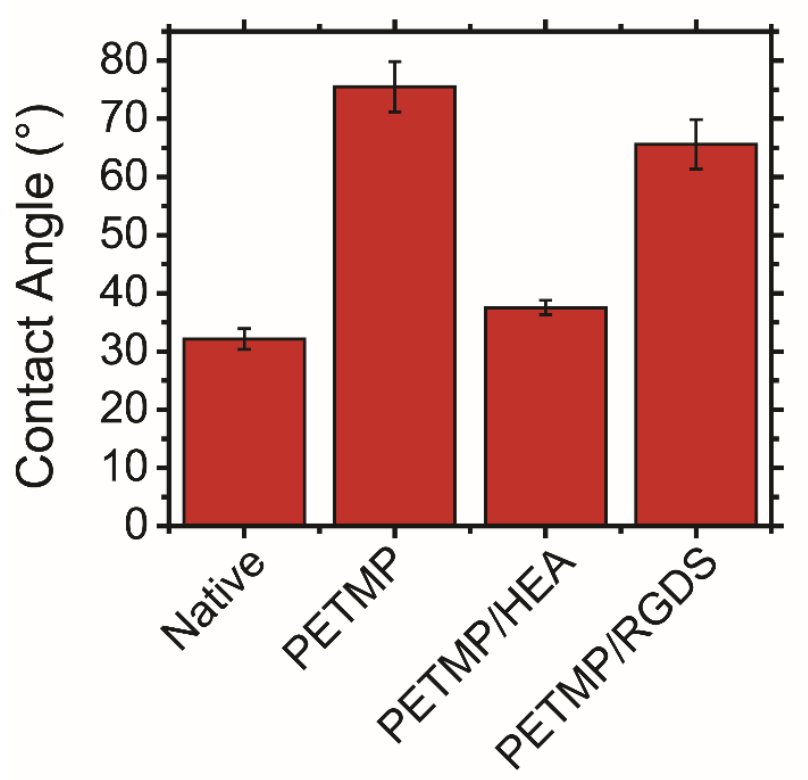

Figure S7. Static Contact angle measurements on flat functionalized elastomer surfaces. Native surfaces exhibited a contact angle of $32 \pm 1^{\circ}$. When functionalized with hydrophobic PETMP, the contact angle rises to $75.5 \pm 4^{\circ}$. With further modification of the thiols with either hydrophilic hydroxyethyl acrylate (HEA) or AF647-RGDS, the contact angle of the surfaces decrease again to $37.5 \pm 1.2^{\circ}$ and $65.6 \pm 4.3^{\circ}$, respectively. All contact angle experiments were conducted on flat substrates which were created with the exact same formulation and experimental conditions as wrinkled samples.

a.
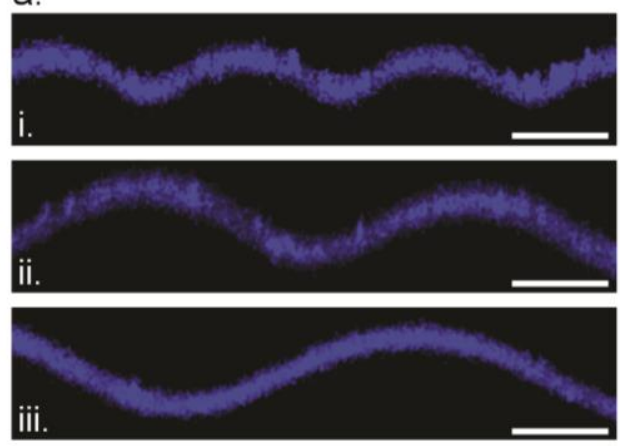

b.

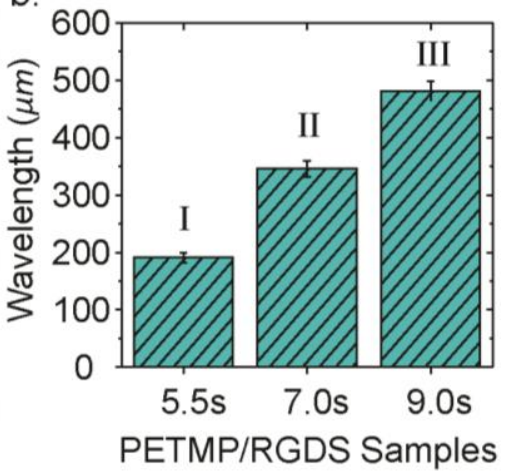

C.

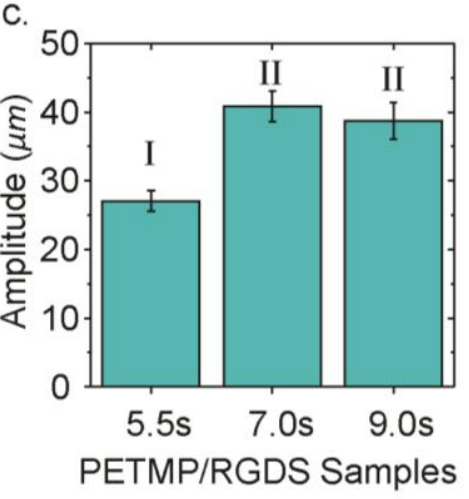

Figure S8. Wavelength and amplitude measurements via confocal microscopy. (a) Cuts through the confocal images capture the wrinkle topography of the substrate surface for short ( 5.5 second irradiation, $i$ ), medium ( 7 second irradiation, ii), and long ( 9 second irradiation, iii) wavelengths. Scale bar $100 \mu \mathrm{m}$. (b) Wavelength measurements from confocal images. (c) Amplitude measurements from confocal images. Roman numerals are statistically the same. 


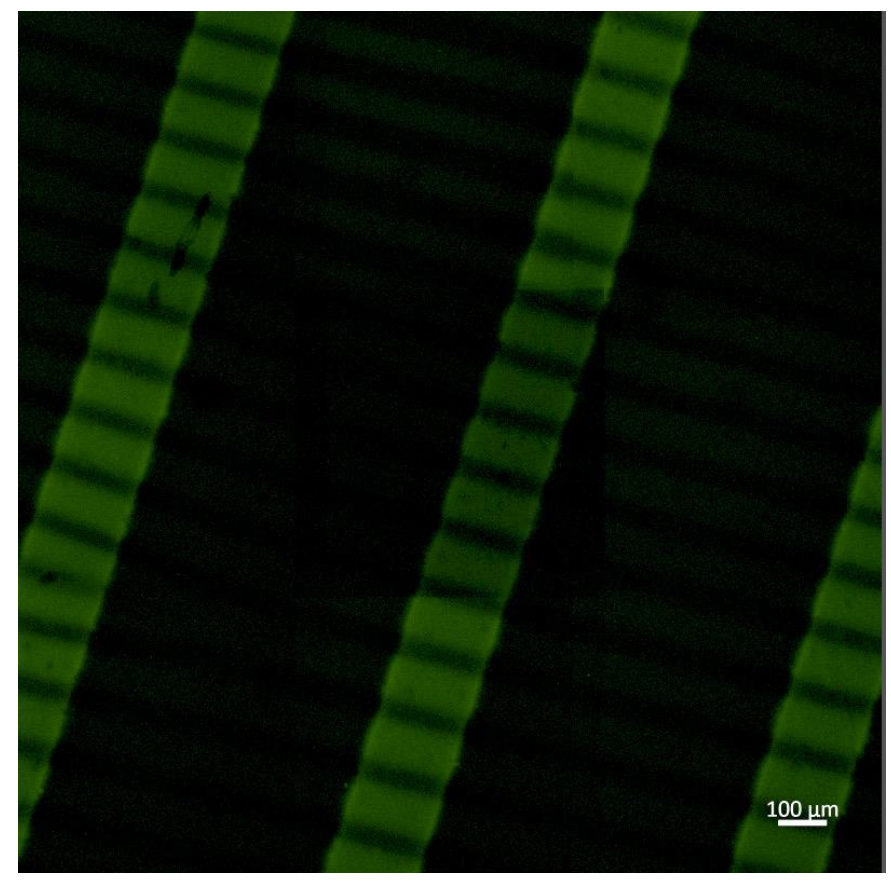

Figure S9. Confocal images of wrinkles photopatterned with fluorescent probe. Top-down image of wrinkles photopatterned with parallel lines using PETMP and fluorescently labelled-RGDS. Wrinkle periodicity is very uniform across the entire sample.

a.

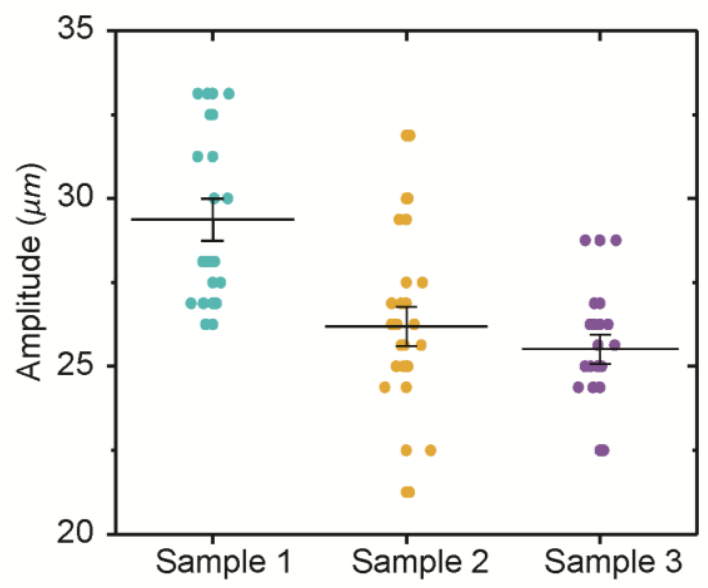

b.

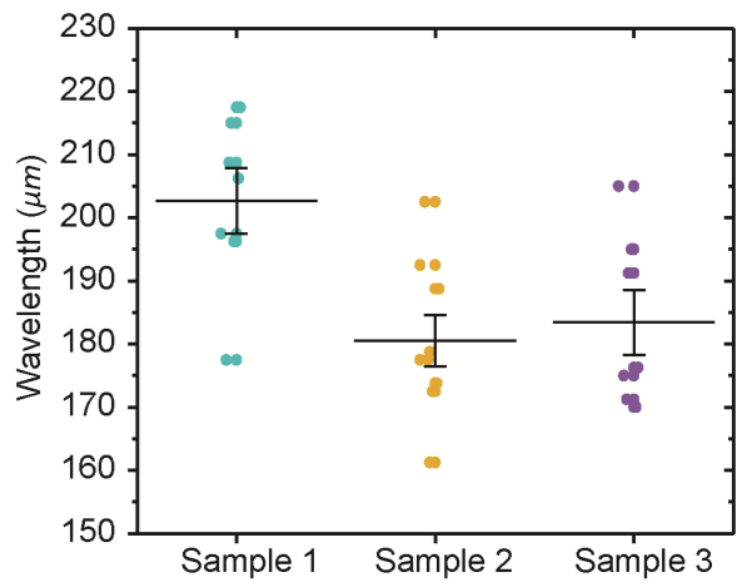

Figure S10. Consistency of amplitude and wavelength across a single sample and between replicates. Confocal images were used to measure amplitudes (a) and wavelengths (b) across images from three replicates of the 5.5 second irradiation condition. For both amplitude and wavelength, measurements were consistent both within a single sample and between different samples. 

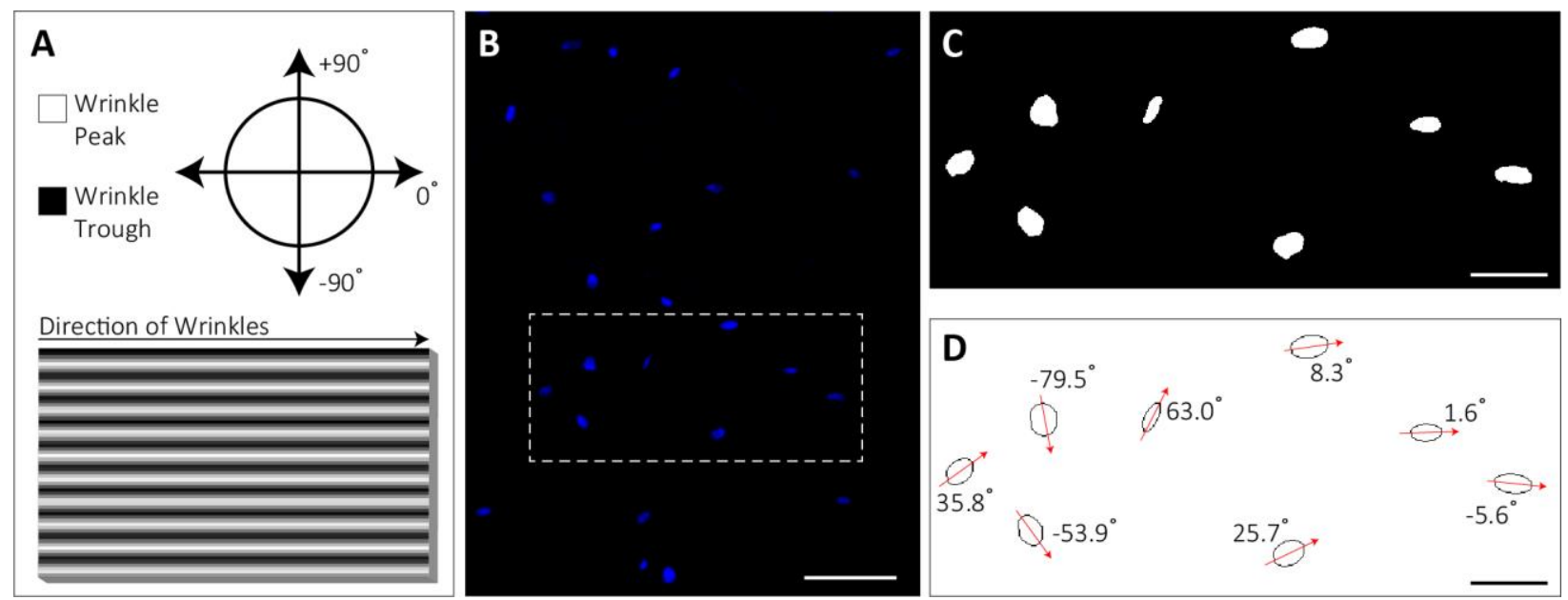

Figure S11. Protocol for determining cellular alignment of hMSCs on wrinkled substrates. (A) The direction of wrinkles was assigned as $0^{\circ}$ and nuclei orientation was determined as the angle relative to the wrinkle direction. (B) Nuclei were stained with DAPI for visualization. Scale bar $100 \mu \mathrm{m}$. (C-D) Nuclei images were processed and each nucleus fitted to an ellipse, from which the angle of orientation was determined. Alignment was taken to be $\pm 15^{\circ}$ off axis from $0^{\circ}$. Scale bar $50 \mu \mathrm{m}$. 
Table S1. Statistical analysis of hMSC attachment in response to surface functionalization (to 3 significant figures).

\begin{tabular}{|c|c|c|c|c|c|}
\hline Area Density & Native & PETMP & PETMP/HEA & PETMP/RGDS & RGDS Only \\
\hline Native & & 0.0696 & 1.00 & $0.000215 * * *$ & $0.00663 * *$ \\
\hline PETMP & $0.00738 * *$ & & 0.0696 & $0.00228 * *$ & 0.665 \\
\hline PETMP/HEA & 1.00 & $0.00738 * *$ & & $0.000215 * * *$ & $0.00663 * *$ \\
\hline PETMP/RGDS & $0.000000402 * * * *$ & $0.00618 * *$ & $0.000000402 * * * *$ & & $0.00287 * *$ \\
\hline RGDS Only & $0.000567 * * *$ & 0.362 & $0.000567 * * *$ & $0.0289 *$ & \\
\hline
\end{tabular}

Table S2. Statistical analysis of hMSC attachment with increasing wrinkle wavelength (to 3 significant figures).

\begin{tabular}{|c|c|c|c|c|}
\hline Area Density & Flat & 5.5 s Wrinkled & 7 s Wrinkled & 9 s Wrinkled \\
\hline Flat & & 0.648 & 0.390 & $0.0413 *$ \\
\hline 5.5 s Wrinkled & $0.0000221 * * * *$ & & 0.728 & 0.146 \\
\hline 7 s Wrinkled & 0.139 & 0.251 & & 0.233 \\
\hline 9 s Wrinkled & $0.0190 *$ & $0.0340 *$ & 0.900 & \\
\hline
\end{tabular}

$$
\begin{array}{r}
* p<0.05 \\
* * p<0.01 \\
* * * p<0.001 \\
* * * * p<0.0001
\end{array}
$$

Table S3. Statistical analysis of hMSC nuclear alignment with increasing wrinkle wavelength (to 3 significant figures).

\begin{tabular}{|c|c|c|c|c|}
\hline $\begin{array}{c}\text { Nuclear } \\
\text { Alignment }\end{array}$ & Flat & $\mathbf{5 . 5}$ s Wrinkled & $\mathbf{7}$ s Wrinkled & $\mathbf{9}$ s Wrinkled \\
\hline Flat & $0.0497 *$ & $0.0361 *$ & $0.00447 * *$ \\
\hline $\mathbf{5 . 5}$ s Wrinkled & & 0.832 & 0.986 & 0.781 \\
\hline $\mathbf{7}$ s Wrinkled & & & $0.05 \%$ \\
\hline $\mathbf{9}$ s Wrinkled & & & \\
\hline
\end{tabular}

